\title{
An Analysis Of Inter-Device Transmission In Wireless Connections
}

\author{
Wycliffe Lamech Ogogo ${ }^{1}$, Meshack Otunge Okong'o ${ }^{2}$, Joseph Singori ${ }^{3}$ \\ ${ }^{1}$ Kisii University, P.O Box 408-40200, Kenya, \\ ${ }^{2}$ Eden University, Lusaka-Zambia, \\ ${ }^{3}$ University of Kabianga, P.O Box 3020-20200, Kenya \\ DOI: 10.29322/IJSRP.11.09.2021.p11719 \\ http://dx.doi.org/10.29322/IJSRP.11.09.2021.p11719
}

\begin{abstract}
It is anticipated that communication between two or more computing devices (Inter-Device) would piece together an important part of any forthcoming wireless link by way of its assurances in providing extremely low latency on behalf of messaging amongst operators. Such innovative approach would possibly function in a spectrum that is either certified or prohibited. Inter Device linkage therefore emerges as a unique addition to the old-fashioned cell phone messaging architype, whose underlying merits are nonetheless, supplemented by many practical and commercial concerns that are essential to a prompt resolution, in advance of assimilating it into a cellular connection. The main aim of this paper is to ascertain some core features of inter device links together with its procedural set-ups, design, practical features and the extent of any other active study. Research papers, Journals, Internet Sites, and books were used to organise the subject's relevant content. The analysis conducted by this study indicates that momentary overview of (ID) communication has been offered above in relations to its functionalities, design and the key technical experiments to its execution.
\end{abstract}

Index Terms $-4^{\text {th }}$ Generation Network, $5^{\text {th }}$ Generation Network (5G), Inter-Device, Long-Term Evolution (LTE), User Gear (UG), Wireless link.

\section{INTRODUCTION}

$\mathrm{A}$ worldwide rollout of the wireless gadget connection currently stands at the $4^{\text {th }}$ generation since its inception in 2009. There has been an honest need for faster multimediagenerous data interchange alongside excellent quality voice calls to primarily arouse this advancing ambition. Fresher applications that are more technically challenging have been developed and there being a potential for an exponential surge in subscriber base, an imperative obligation is fronted for an innovative technique to enhance data rates while latency stays at its bare minimum. Inter Device linking is an innovative model in wireless linkage which permits User Gear (UG) within visible distances to interconnect via a direct link instead of their wireless signals drifting over to the base station (BS).

The ID model supports an extremely low latency when messaging through the implementation of a smaller signal passage. Several short-range wireless technologies that are demarcated by the 3rd Generation Partnership Project (3GPP) such as Bluetooth, LTE Direct and Wireless Fidelity Direct, may be harnessed to boost Communication through (ID) as they only contrast typically in distance within 1-hop gadgets, the data-rates, mechanisms used for gadget detection and classic applications. For example, the Bluetooth, can hold a data rate determined at $50 \mathrm{Mbps}$ and a close range of up to 240 meters, Wi-Fi Direct permits range to a rate of $250 \mathrm{Mbps}$ and 200 meters whereas LTE Direct provides up to $13.5 \mathrm{Mbps}$ together with a range of 500meters (Haus, et al.,2017). Inter Device link would therefore make operators to be extra flexible when it comes to discharging traffic flow on the main network, improving the effectiveness of spectral and lowering the cost per bit and energy used.

The figure below represents both the Cellular and Inter Device Communication Single and Multi- hop and network moulding by Inter Device connections.

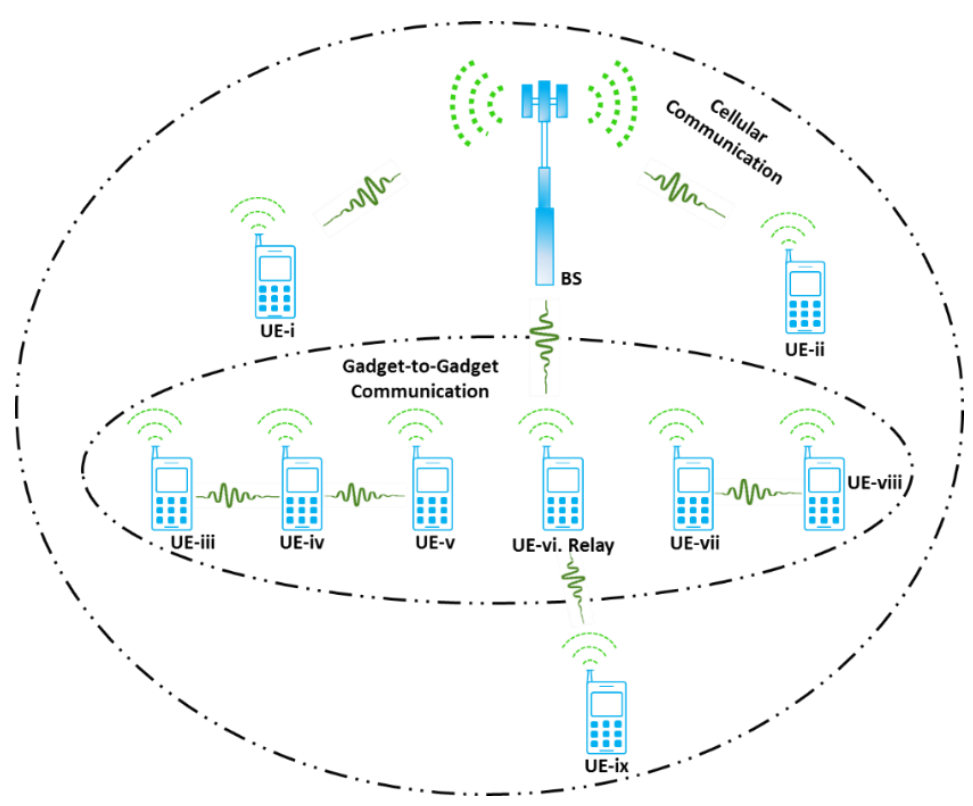

\section{Figure I: Cellular \& (ID) Communication; Single-hop \&} Multi -hop and Network Moulding

Figure 1 represents the Inter Device and cellular communication which represent both the single and multi-hop networks moulded by ID connections. 


\section{DISCUSSION OF KEY FACTORS IN INTER-DEVICE TRANSMISSION IN WIRELESS CONNECTIONS}

In examining inter-device transmission in wireless connections, various factors were evaluated.

\section{Usage of Inter Device Communications}

Communicating using ID allows transfer of huge data volumes speedily between devices that are moveable within a short range. It is further established that some of the most prominent situations where Communication via ID has been a practise has been found to be effective.

\section{Local Data Facilities}

Inter Device communication is able to sustenance the local data facilities quite competently through (one-to-one) unicast, (one-to-several) multicast and (one-to-all) broadcast transmissions. These application examples comprise and not limited to the following;

\section{Information Sharing}

User Gears (UG's) can influence Inter Device connection to move data in whichever format with inferior energy and greater data rates than in predictable cellular networks. They can enable efficient streaming facilities similar to Google's Chromecast, IPTelevision and Over-the-top media services. This is realised when the UGs form bands that cast data inside a cluster. They likewise offer support in additional services within the vicinity, for example when there is a threat to the safety of the public. Networks of Inter Device are able to operate unhampered in hot-zone where within range all base station are grounded.

\section{Data and Computing Off-Load}

A gadget with an efficient and excellent connectivity is able to operate as a hotspot with cached data from the BS and any different tool which has the ability to download data by means of Inter Device connection. UGs with deprived processing power or sometimes little energy funding may somewhat off-load heavy computation workload to UGs close by using ID connection, being considered to have a better capability. Aijaz, et al, (2013) assert that a substantial amount of research has been directed to the design of the off-loading practice and this can further be identified from.

\section{Expansion of Coverage}

When a User Gear is implemented at the perimeter of a cell or in an area hit by a calamity may stumble upon week signal quality during connection to the base station. If at all there is a UG which in this paper are $(\mathrm{Y})$ with a better linkage to the base station, it would then act like a relay for that matter, and as a result a the Inter Device Connection $\mathrm{X}-\mathrm{Y}$ than is tagged by a cellular connection Y - base station would attach to the BS. Relays are used to spread the coverage of cell service and to enable multi-hop communication. An additional approach to boosting signal power at a recipient is to transmit it by means of multiple corresponding paths, each one composed of a reciprocated appliance. These procedures are regularly referred to as cooperative diversity procedures. A macro-cell tier consisting of BS-to-Gadget communication and a Gadget tier comprising inter device communication can be traced back in the adjacent link, (Tehrani, et al.,2014).

\section{Inter-Device (ID) Contact}

Inter Device contact in this case enables piece of technology implemented for Internet-of-Things (IoT). Inter Device contacts involve independent connectivity and contact surrounded by devices that range from fixed low-power machines to powerful ones. Inter Device linkages launch Inter Device communication especially in IoT because of the ultra-low latency affordability required for the same, hence; ensures a concurrent response whenever required, (Bello \& Zeadally, 2016). One such application is (V.2.V) communication where contact using Inter Device can be exploited for sharing information between neighbouring motor vehicles swiftly as well as to off-load traffic jams efficiently. They can also be exploited for motor vehicle-toinfrastructure and motor vehicle-to-pedestrian contact.

\section{Architecture Spectrum/Field Distribution}

According to Asadi, (2014), the Inter Device spectrum is widely categorised into two different types of fields; this can be either in or out band.

\section{In-Band Inter Device Communication}

When this type of communication is engaged, both the cellular and the Inter Device link use matching spectrum that is licensed to that particular cellular operator. This spectrum can either be sub-divided into an Inter Device) non-overlapping lot and cellular link in that order, otherwise referred to as an (overlay) or it sometimes may not be separated at all, thereby referred to as an (underlay). An Overlay system is easier to implement while an underlay structure would lead to an opportunistic scenario hence, a more competent spectrum usage and better turnover to its operators.

\section{Out-Band (ID) Communication}

When implementing the Inter Device communication within an unrestricted spectrum, for instance a $2.4 \mathrm{GHz}$ ISM band which is free or the Wave band of $38 \mathrm{GHz} \mathrm{mm}$ where the mobile linkage doesn't transpire. Out-band field distribution aids in the elimination of the intrusion among the (ID) and cellular consumers even though there is still an interference that is active in another electronic gadget such as (Wireless Fidelity \& Bluetooth) which are known to operate in this band. Actually, the operatives can be able to regulate interference when using an approved spectrum but, that is not likely for the out-band structure. The Out-band expertise is additionally distributed into autonomous and controlled categories. In discussion about the controlled set, communication in ID its radio interface is measured using cellular link as compared to autonomous set, which connection in cellular controls are limited to the communication of the cellular only, separate from the communication of ID control which the it is managed by users.

\section{Inter Device Communication in LTE-Advanced}

In LTE-Advanced standard, 3GPP Rel. 12 requires a universal conception of proximity-based services (ProSe) which permit tangibly close gadgets to notice themselves and interconnect by way of direct linkage, (Lien, et al, 2016). ProSe is predestined for safety communication of the public besides commercial use. ID communication and discovery is demarcated as ProSe support as It is similarly referred to as Direct LTE for supporting direct complete communication between the UGs that use certified spectrum \& the LTE global ecosystem.

An extremely prototypical that is made simple for ID way of communication established on ProSe's architecture. The eNB 'as it's referred to in 3GPP' or BS is linked to the Evolved Packet Core (EPC) which then converses with the UG in a straight line using cellular linkage. In addition, UGs can converse via direct ID 
contacts. As far as of channel structures are concerned, a direct connection between two UGs is referred to as side-link as it is able to function by time division and also frequency division duplex. Upon powering up, UGs would at the outset synchronize with the
eNB or other UGs within range and for this purpose, quite a number of synchronization signals end up as explained in the 3GPP Rel.12 (Lien, et al., (2016).

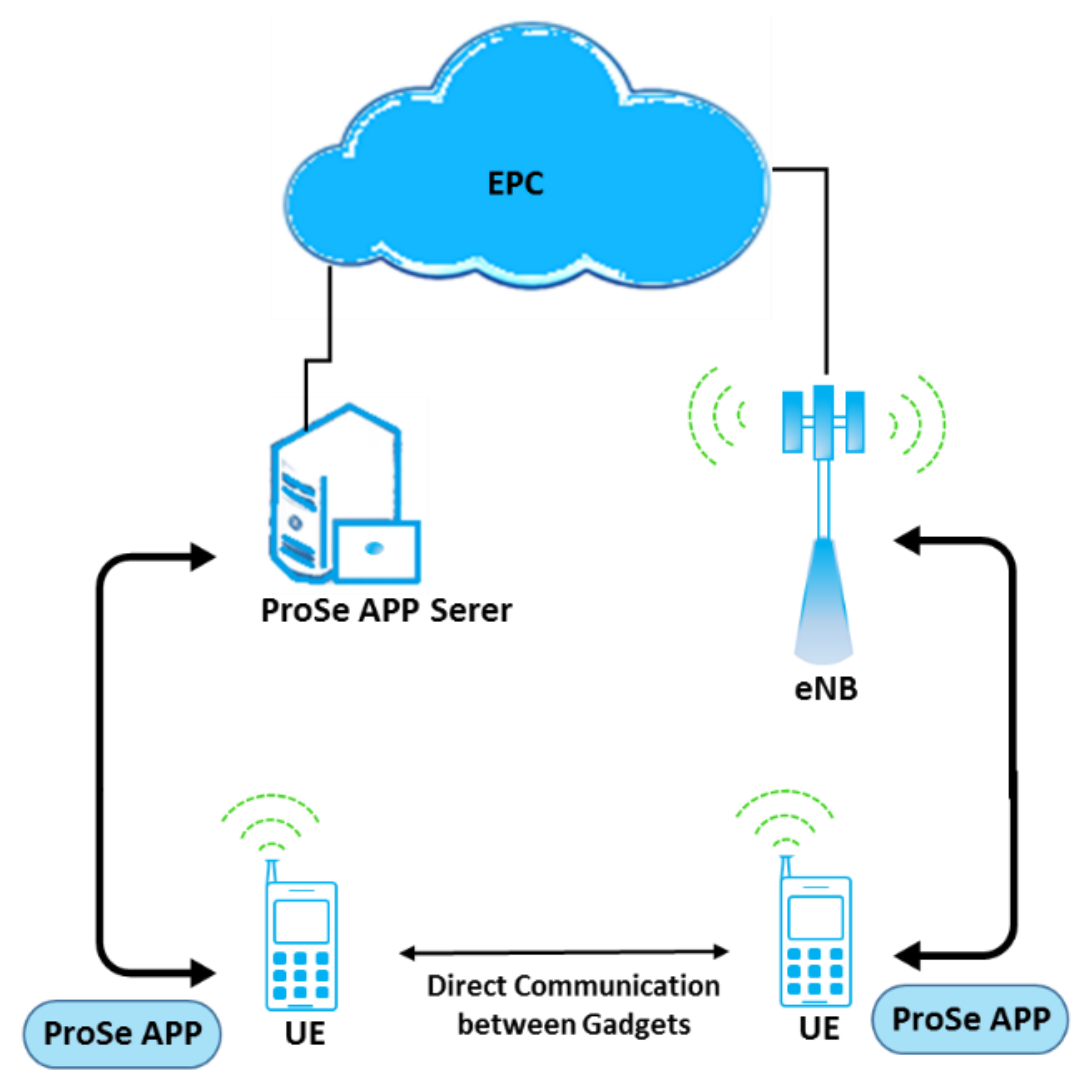

Figure 2: Basic ID Communication on ProSe architecture

\section{Single-Hop and Multi-Hop Linkage}

Mostly an ID connection bonds with a UG transmitter and have it projected over the UG receiver that finally sends communication that is single-hop. A link that's multi-hop comprises of ID connections that are evocative of the mobile ad hoc network (MANET) a mobile ad hoc network where the intermediary UGs performs as relays either sandwiched in between a UG and a BS or in between 2 UGs. A variation of initial set-up may perhaps be the cooperating cluster of a UGs where data files are transferred by BS to cluster head that in turn group-casts it to another UGs of the same cluster. The 3GPP Rel. 12 empowers the UG-to-network transmit whereas 3GPP Rel. 12 advances maintenance for communication that is vehicular that is to say, high density and also high speed of the nodes grounded on ID proficiency.

\section{CHALLENGES OF INTER DEVICE COMMUNICATION}

In the introductory aspect of this section, we resolve to deliberate on the several practical aspects and resultant problems of ID linkages in networks that is wireless.

\section{Harmonisation}

In distinctive cellular network, the UGs realize both time and frequency harmonization by use of using broadcast that are periodic which originate from BS. Gadgets using ID communication may harmonize also of similar broadcasts as in turn they are of the similar BS. Circumstances becomes complex in subsequent scenarios as here: (i) UGs fit in non-similar BSs that may not harmonized by themselves, (ii) some of UGs are within the radius or some out of range of the network, and (iii) all UGs lies exterior to the coverage network, (Abedini, et al.,2016). Harmonization in between UGs is advantageous for (ID) linkage since it supports a UG to utilise the right frequency and also timeslot for discerning and to communicate with its own peer, eventually participates in a messaging that is of extra energyefficient (Sun, et al., 2017).

\section{Peer Detection}

The view of demand for ID link, suggests there ought to be a well-organized means of discerning peers. A UG should be capable of detecting other UGs within vicinity as swiftly and uses minimal power. From the user standpoint, there exists 2 kinds of peer detection methods, open \& restricted, (Feng, et al., 2014). In accordance to the first instance, gadgets can't be detected by final user who without their approvals. In second instance it is the actual opposite where gadgets are visible to other users whenever they lie within proximity. From the network position, peer findings can 
be well-ordered tightly or maybe lightly by BS, (Feng, et al.,2014). In a multicellular grid, it's fairly problematic to be assisted from neighbouring BSs, allowing a peer to detect a job that's challenging, (Gandotra, \& Jha 2016). Incentive-based arrangements might be scrutinized as a feasible answer to this hurdle.

\section{Approach selection}

User groups that are in pairs which have themselves detected are possible nominees for a communication of ID. Nonetheless, in terms of performance, cellular communication might be a better supplementary for instance, if noisier is the direct channel. Selection of mode is concerned with choosing the mode which is the right - ID or cellular - for the communication in between 2 UGs to attain performance objective as the following; low transmitting power low latency, low transmit power, or high spectral efficiency. The link itself can do an approach selection or it can be left for the UGs. To convey the approach selection difficulty, an individual may perhaps relate a judgement variable with every UG, which captures the nominated approach and eventually then supplement a diversity of intentions \& restrictions. A modest intention might be the gained channel of the nominated approach ought to be greater than that of other possible approaches. Additional refined intentions like peak reuse spectrum, etcetera, weighted-sum-rate maximization, might in turn be considered for implementation. Restrictions might be least QoS at the maximum transmitting power, end receiver, among others. Therefore, selection approach is in general combined with control of power. The study could be completed by use of immediate system material [might be a tough sell acquiring in practice] or a system that is statistical having evidence that leads to conclusions in which corresponds optimally at a certain instant or maybe optimal in ordinary logic over an extended period, (El Sawy, et al., 2014).

\section{Resource Distribution}

A subcarrier, which is arguably a good example of a radio resource is a significant step in the in-band methodology where formation and sustenance of direct relations between pairs of Inter Device in cellular setup is allocated. Lin, et al., (2014). Proposes a simplified yet universal resource distribution structure for inband multicellular scheme in uplink spectrum Overlay is spread into 2 orthogonal segments with portion $\eta$ allocated to Inter Device \& 1- $\eta$ to cell messaging; in underlayment, the field is disseminated into $\mathrm{B}$ bands and Inter Device user groups can arbitrarily and unconventionally access $\beta B(\beta \in[0,1])$ of each. The peak values found in $\beta \& \eta$ are calculated by presuming that user groups are dispersed in accordance to an indiscriminate point process of spatial Poisson and selection approach is grounded on a user groups' proximity to its anticipated end receiver, of which then boosting performance objective that includes a joint occupation of rates attained by a cellular and prospective user of Inter Device. Diverse distribution of resource schemes may be deliberated by fluctuating the tallying several restrictions and objectives optimization.

\section{Interference Handling}

In in-band communication method, both the cellular \& Inter Device contacts can restrict one other based upon the way their frequencies are shared. In the out-band communication, an Inter
Device contacts incur interference beginning from one other besides additional gadgets that operate in the same band, Noura and Nordin, (2016). When the UGs transmit at an inferior power level, which may or may not disturb the QoS at the end receiver, interference can however be reduced. Thus, awareness of interference resource administration is an intricate optimization challenge. Regularly it's cast as a problem of weighted-sum-rate maximization that is subject to higher transmission strength and lower QoS restrictions or as a problem of transmit power minimization that is subject to the smallest QoS restriction, (Ali and Ahmad, 2017).

Power control, adding to interference easing, and points to an energy-efficient procedure which is one of the objectives of wireless networks of the next generation. Cautious scheduling of broadcasts also offers assistances to lessen interference. Appropriate coding schemes \& modulation (selected referred on quality of channel) \& hybrid automatic repeat request (a blend of Forward Error Correction - FEC) \& Automatic Repeat Request $\mathrm{ARR}$ ) rises the strength of the conveyed signal in contrast to noise. Interference minimization, method selection, resource distributions are closely associated and are optimized frequently jointly. For the 3 complications, a number of hybrids, centralized, and distributed algorithms have since been suggested but the jury is still out on the same, (Ali and Ahmad, 2017).

\section{Inter Device Flexibility}

Similar majority of the studies on most Inter Device have concentrated on users that are static whereas cellular setups fundamentally accommodate mobile users. Further analysis is necessary in order to appreciate the way performance advances auger in scenarios that are dynamic and which handover mechanisms and interference management are desirable as UGs traverse cells, Murkaz, et al., (2016). Multi-hop Inter Device communication has also posed several challenges, Wei, et al., (2016).

\section{Pricing}

Pricing is a key persistent issue to cellular service providers. The challenging query is a way to regulate the undeviating link among the gadgets and methods to bill the subscribers. Countless models of pricing have been expounded by Tehrani, et al. (2014) suggesting that cellular operators may decide to use UGs as transmitter for other subscribers and might offer them monetary enticements to the relay UGs. Service providers can also correspondingly offer billable services such as safety during the ID communication. Other cost-effective representations in the perspective of ID communication involve the way ID UGs in cluster may transact data files. According to Wang, et al (2016), cellular service subscribers can vend their bandwidth to (ID) UGs and also ID -capable UG pairs can auction their cell assets to other future cellular service subscribers as they themselves go for (ID) communication (Li, Guo and Stojmenovic, 2016).

\section{Security}

Inter Device communication manages a resilient privacy and data discretion likened to conventional cellular linkage as data is not kept at a central site. Nonetheless, several mutual attacks such as denial of service, malware attack, eavesdropping, man-in-themiddle, device IP spoofing and many others may paralyse Inter Device exchanges. Subscribers would also love to safeguard their confidentiality, for example by limiting the accessibility of most of their delicate private information. Absence of a principal 
authority in such a communication setup makes it very challenging to implement safety and discretion procedures. Haus, et al., (2017) has a model that illustrate in 3-dimensional space the following threats: (1) whether invader is an insider or outsider, (2) or whether an assailant is online for example if it alters in-transit information or passive when it snoops only on file data and (3) whether the inversion is resident or extended transversely throughout the grid. A number of suggestions have been made as a safety net to the Inter Device networks as reviewed by Haus, et al., (2017) and Gandotra, Jha and Jain (2017).

\section{INTER DEVICE COMMUNICATION AND 5G LINKAGES}

The imminent setup of $5 \mathrm{G}$ is anticipated to convey data rates that are aggregated (like, complete volume of data that a network is able to attend, and its measurements is in bits/sec/area) 1000 times to the existing 4G setup, Wang, (2014). The spectral effectiveness \& efficiency of energy would be stretches greater than of a 4G linkage, data-rates for cellular subscribers would be end-to-end latencies \& multi-Gbps of about $1 \mathrm{~ms}$. The $5 \mathrm{G}$ setup would in essence comprise a canopy of technologies that include heterogeneous network which involves usage of several radio accessing technologies, various backhaul techniques $\&$ a pyramid of pico, femto, cells-macro and enormous MIMO (that is a huge antenna arrays at BS installed to attend countless subscribers simultaneously), CRN (cognitive radio network) [where minor / tertiary subscribers opportunistically tap the spectrum of a primary users], mmWave (millimetre wave) spectrum [operates at above 30-300 GHz frequency] \& the (ID) communication, Wang, C. (2014).

The last option enlisted above is primarily significant in $5 \mathrm{G}$ in spite of the challenges of implementation, Tehrani, et al., (2014). The spectrum of mmWave embedded in 5G may be utilised to create a short-range (ID) relation among UGs. Subsequently mmWave experiences small multi-user intrusion, lots of mmWave. Inter Device networks may work simultaneously, thus enhancing network capability. Subordinate operators in a CRN may in turn use an Inter Device connection as to circumvent interference caused to main users. Inter Device links complements massive-MIMO-facilitated \& HetNets BSs in enlightening rates of data $\&$ spectral effectiveness. Also, MIMO embedded antennas in UGs would upsurge noise resilience \& structure size by using multiplexing and diversity gains. Communication of (ID) -with relays of MIMO-enabled gadgets may also boost system size considerably (Asadi, 2014).

\section{CONCLUSION}

A momentary overview of Inter Device communication has been discussed in relations to its functionalities, design and the key technical experiments to its execution. The Inter Device linkage can also be an essential part in comprehending the aspiring objectives of the $5 \mathrm{G}$ contactless networks. Initial structures that employed the Inter Device communication have been found to be effective. Additional expansions and industry standards are also progressively being set up.

\section{REFERENCES}

[1] 3GPP, 3rd generation partnership project (3GPP). Available: http://www.3gpp.org/ (Accessed 01 February 2020). Google Scholar

[2] Abedini N., Tavildar S., Li J., Richardson T.Distributed synchronization for device-to-device communications in an lte network, IEEE Trans. Wireless Commun., 15 (2) (2016), pp. 1547-1561. CrossRefView Record in ScopusGoogle Scholar

[3] Aijaz, A., Aghvami, H., \& Amani, M. (2013). A survey on mobile data offloading: technical and business perspectives. IEEE Wireless Communications, 20(2), 104-112.

[4] Ali, S., \& Ahmad, A. (2017). Resource allocation, interference management, and mode selection in device-to-device communication: a survey. Transactions on Emerging Telecommunications Technologies, 28(7), e3148.

[5] Asadi, A., Wang, Q., \& Mancuso, V. (2014). A survey on device-to-device communication in cellular networks. IEEE Communications Surveys \& Tutorials, 16(4), 1801-1819.

[6] Bello, O., \& Zeadally, S. (2014). Intelligent device-to-device communication in the internet of things. IEEE Systems Journal, 10(3), 1172-1182.

[7] ElSawy, H., Hossain, E., \& Alouini, M. S. (2014). Analytical modeling of mode selection and power control for underlay ID communication in cellular networks. IEEE Transactions on Communications, 62(11), 4147-4161

[8] Kar, U. N., \& Sanyal, D. K. (2020). A Critical Review of 3GPP Standardization of Device-to-Device Communication in Cellular Networks. SN Computer Science, 1(1), 37

[9] Haus, M., Waqas, M., Ding, A. Y., Li, Y., Tarkoma, S., \& Ott, J. (2017) Security and privacy in device-to-device (ID) communication: A review. IEEE Communications Surveys \& Tutorials, 19(2), 1054-1079.

[10] IEEE ComSoc, (2021) Best reading topics on device-to-device communications. Available: http://www.comsoc.org/bestreadings/topics/device-device-communications (Accessed 03 February 2020).

[11] IEEE Trans. Veh. Technol., 65 (9) (2016), pp. 7476-7487.

[12] J. Netw. Comput. Appl., 71 (2016), pp. 99-117. Article: Downloaded PDFView

[13] Kebriaei, H., Maham, B., \& Niyato, D. (2015). Double-sided bandwidthauction game for cognitive device-to-device communication in cellular networks. IEEE Transactions on Vehicular Technology, 65(9), 7476-7487.

[14] Li, P., Guo, S., \& Stojmenovic, I. (2015). A truthful double auction for device-to-device communications in cellular networks. IEEE Journal on Selected Areas in Communications, 34(1), 71-81

[15] Lien, S. Y., Chien, C. C., Tseng, F. M., \& Ho, T. C. (2016). 3GPP device-todevice communications for beyond $4 \mathrm{G}$ cellular networks. IEEE Communications Magazine, 54(3), 29-35.

[16] Lin, X., Andrews, J. G., \& Ghosh, A. (2014). Spectrum sharing for deviceto-device communication in cellular networks. IEEE Transactions on Wireless Communications, 13(12), 6727-6740.

[17] Murkaz, A., Hussain, R., Hasan, S. F., Chung, M. Y., Seet, B. C., Chong, P H. J., ... \& Malik, S. A. (2016, August). Architecture and protocols for intercell device-to-device communication in 5G networks. In 2016 IEEE 14th Int Conf on Dependable, Autonomic and Secure Computing, 14th Intl Conf on Pervasive Intelligence and Computing, 2nd Intl Conf on Big Data Intelligence and Computing and Cyber Science and Technology Congress (DASC/PiCom/DataCom/CyberSciTech) (pp. 489-492). IEEE.

[18] Noura, M., \& Nordin, R. (2016). A survey on interference management for device-to-device (ID) communication and its challenges in 5G networks. Journal of Network and Computer Applications, 71, 130-150.

[19] Sun, W., Brännström, F., \& Ström, E. G. (2016). Network synchronization for mobile device-to-device systems. IEEE Transactions on Communications, 65(3), 1193-1206.

[20] Tehrani, M. N., Uysal, M., \& Yanikomeroglu, H. (2014). Device-to-device communication in $5 \mathrm{G}$ cellular networks: challenges, solutions, and future directions. IEEE Communications Magazine, 52(5), 86-92.

[21] Wang, C. X., Haider, F., Gao, X., You, X. H., Yang, Y., Yuan, D., ... \& Hepsaydir, E. (2014). Cellular architecture and key technologies for 5G wireless communication networks. IEEE communications magazine, 52(2), 122-130.

[22] Wang, J., Jiang, C., Bie, Z., Quek, T. Q., \& Ren, Y. (2016). Mobile data transactions in device-to-device communication networks: Pricing and auction. IEEE Wireless Communications Letters, 5(3), 300-303. 
[23] Wei, L., Hu, R. Q., Qian, Y., \& Wu, G. (2015). Energy efficiency and spectrum efficiency of multihop device-to-device communications underlaying cellular networks. IEEE Transactions on Vehicular Technology, 65(1), 367-380.

\section{AUTHORS}

First Author - Wycliffe Lamech Ogogo, Kisii University, P.O Box 408-40200, Kenya, Email: pricelamech@ gmail.com

Second Author - Meshack Otunge Okong’o, Eden University, Lusaka-Zambia, Email: meshackokongo@gmail.com

Third Author - Joseph Singori, University of Kabianga, P.O Box 3020-20200, Kenya. Email: josmo841@ gmail.com 